\title{
Chronic Salpingitis
}

National Cancer Institute

\section{Source}

National Cancer Institute. Chronic Salpingitis. NCI Thesaurus. Code C40118.

Chronic inflammation of the fallopian tube. It usually follows an acute inflammatory attack. 